\title{
Insights on Structuration of Peroxotungstic Acid in Aqueous Media
}

\author{
Julia C. O. Pazinato, ${ }^{a}$ Marcos A. Villetti, ${ }^{b}$ Omar Mertins, ${ }^{c}$ Emerson R. Silva ${ }^{\circledR c}$ and \\ Irene T. S. Garcia ${ }^{\circledR} *, a$ \\ ${ }^{a}$ Departamento de Físico-Química, Instituto de Química, Universidade Federal do Rio Grande do Sul, \\ 91501-970 Porto Alegre-RS, Brazil \\ ${ }^{b}$ Departamento Física, Centro de Ciências Naturais e Exatas, Universidade Federal de Santa Maria, \\ 97105-900 Santa Maria-RS, Brazil \\ 'Departamento de Biofísica, Escola Paulista de Medicina, Universidade Federal de São Paulo, \\ 04023-062 São Paulo-SP, Brazil
}

\begin{abstract}
Recent studies on tungsten oxide preparation are mainly motivated by its applications in smart windows, energy harvesting and, recently, in cancer therapy. Peroxotungstic acid (PTA) is an important precursor to obtain tungsten oxide in an environmentally-friendly way, through the colloidal synthesis in aqueous media. Despite giving chemically pure products, PTA originates oxides with various morphologies depending on the experimental conditions, a subject open to discussion. PTA, in the presence of sodium dodecyl sulfate (SDS) as architecture-directing agent, was investigated in ethanol/water medium by small angle X-ray scattering (SAXS), fluorescence, surface tension and conductivity measurements; the morphology of tungsten oxide obtained from the precursor systems was also characterized by scanning electron microscopy (SEM). PTA/SDS/ethanol/water systems show a two-level organization in which small micelles are aggregated as fractal structures. There is an inverse correlation between gyration radius of the fractal structures of the PTA/SDS systems and particle size of the tungsten oxide films. The interaction of PTA with SDS is observed by reducing the Gibbs energy of micellization; also, it is evidenced that the supressive effect of PTA on pyrene emission is overrided in presence of SDS.
\end{abstract}

Keywords: colloidal synthesis, architecture-directing agent, fractal structure, transition metal oxides

\section{Introduction}

Tungsten oxide, an n-type semiconductor that presents electrochromic and photochromic properties, has drawn attention as material for light harvesting, due to its band gap situated, in general, in the visible part of the electromagnetic spectrum. Recent research has also shown that it is able to control hydrogen peroxide release, being proposed as a key material for cancer therapy. ${ }^{1}$

This material can be obtained through different physical and chemical processes. ${ }^{2-6}$ Chemical methods, such as electrodeposition, ${ }^{2}$ anodization, ${ }^{3,4}$ solvothermal, ${ }^{5}$ precipitation and sol-gel ${ }^{6,7}$ are flexible and permit exploration of various precursors and conditions to obtain tungsten oxide with different compositions, crystal structures and morphologies. ${ }^{1,6,7}$ Precipitation and sol-gel

*e-mail: irene.garcia@ufrgs.br are interesting methods, as they are not expensive and do not require any sophisticated equipment. ${ }^{8,9}$ However, the properties of the final product are very sensitive to temperature, solvent system and also to the presence of architecture-directing agents, as small ligands, polymers and surfactants. ${ }^{10-13}$

Peroxotungstic acid (PTA) is an important precursor and can be used in aqueous media to prepare tungsten oxide, in powder or film forms, eliminating the presence of toxic solvents. PTA is prepared in situ by reacting hydrogen peroxide with tungsten ${ }^{14}$ or tungstic acid. ${ }^{15}$

Studies concerning the use of this precursor, in liquid phase, in presence of structuring agents, have been focused on final properties of the tungsten oxide. ${ }^{5,6,13}$ Sodium dodecyl sulfate (SDS) has been explored as structuring agent in sol-gel, hydrothermal or electrochemical synthesis of tungsten oxide with special focus on the properties of tungsten oxide, mainly electrochromic 
and photocatalytic. ${ }^{16,17}$ However, there have been little discussion addressing the oxide precursors structuration in liquid phase like Salmaoui et al. ${ }^{18}$ work. The authors studied the effect of structuring agent hydrocarbon chain length on the morphology of the resulting oxide.

The structuring process in other materials, as polymers and ionic liquids, is usually studied in solution, ${ }^{19,20}$ however, in material science there is still a lack of knowledge about the behavior of precursor/surfactant in the solvent system, especially PTA and SDS.

This work addresses the synthesis of tungsten oxide films by characterizing the structuration of PTA/SDS in ethanol/water media. Ethanol was added to improve the affinity between the apolar part of SDS and water. This study was performed under controlled conditions of ionic strength and temperature and aims to discuss the relationship between the behavior in soft media and the morphology of the calcined films.

\section{Experimental}

Materials

Tungsten powder (99.9\%, Sigma-Aldrich) and hydrogen peroxide ( $29 \%$, Synth) were used to prepare the precursor system. The solvent and structuring system was composed of ethyl alcohol (99.5\%, Synth), sodium chloride (99.5\%, Fmaia) and SDS (90.0\%, Synth). Silicon (100), covered with a $50 \mathrm{~nm}$ layer of $\mathrm{SiO}_{2}\left(\mathrm{SiO}_{2} / \mathrm{Si}\right)$, was used as substrate for tungsten oxide films.

\section{Precursor system preparation}

Three systems were prepared to clearly identify the effect of the media on PTA structuration in the liquid phase: (i) PTA/SDS/ $\mathrm{NaCl} /$ ethanol/water with $10 \mathrm{mmol} \mathrm{L}-1$ PTA; (ii) SDS/NaCl/ethanol/water; (iii) SDS/ethanol/water. The SDS concentration varied in each system, from 0.005 to $90 \mathrm{mmol} \mathrm{L}^{-1}$.

PTA was prepared by reacting $2.2980 \mathrm{~g}$ tungsten powder with $30 \mathrm{~mL} \mathrm{H} \mathrm{H}_{2}(29 \% \mathrm{v} / \mathrm{v})$. The reaction was conducted in an ice bath, due to its strong exothermic behavior, by adding small amounts of powder to peroxide solution under stirring for $3 \mathrm{~h}$. This solution remained at rest for $24 \mathrm{~h}$ to degrade the excess of hydrogen peroxide after which, $2.9220 \mathrm{~g} \mathrm{NaCl}$ was added and the final volume $(250 \mathrm{~mL})$ completed with a mixture of ethanol/ Milli-Q water (1:3 v/v) under stirring $(2 \mathrm{~h})$. The final concentration was $50 \mathrm{mmol} \mathrm{L}^{-1}$ PTA and $200 \mathrm{mmol} \mathrm{L}^{-1}$ $\mathrm{NaCl}$ (stock solution A).

SDS stock solution (B) was prepared by dissolving
$14.4190 \mathrm{~g} \mathrm{SDS}$ and $2.9200 \mathrm{~g} \mathrm{NaCl}$ in $250 \mathrm{~mL}$ of ethanol/ Milli-Q water (1:3 v/v) under stirring. This solution was stirred for $3 \mathrm{~h}$ and remained at rest for $24 \mathrm{~h}$. The final concentration was $180 \mathrm{mmol} \mathrm{L}^{-1} \mathrm{SDS}$ and $200 \mathrm{mmol} \mathrm{L}^{-1}$ $\mathrm{NaCl}$. The diluted systems were prepared by adding to the stock solution $200 \mathrm{mmol} \mathrm{L}{ }^{-1} \mathrm{NaCl} /$ ethanol/water mixture. Salt was added to keep the ionic strength constant.

\section{Characterization of the aqueous system}

The systems were characterized by fluorescence spectroscopy, small angle X-ray scattering (SAXS), $\mathrm{pH}$, electrical conductivity and surface tension measurements.

The surface tension measurements were performed by the Du Nöuy method, at $25^{\circ} \mathrm{C}$, in a Krüss GmbH K20 Easy Dyne tensiometer connected to a Julabo F12 thermostat.

The $\mathrm{pH}$ was measured in a pHmeter DM-20 (Digimed) and the conductivity measurements in an ION conductivity meter, model DDS-12DW. All measurements were performed at $25^{\circ} \mathrm{C}$.

The structure of the SDS/ $\mathrm{NaCl} /$ ethanol/water, as well as PTA/SDS/NaCl/ethanol/water, was evaluated by SAXS. The essays were carried out on an SAXS 1 beamline at Laboratório Nacional de Luz Síncrotron (LNLS, Campinas, Brazil). About $300 \mu \mathrm{L}$ of SDS/PTA solutions were loaded into liquid cells composed of mica windows separated by a path length of $1 \mathrm{~mm}$. X-rays with $\lambda=0.154 \mathrm{~nm}$ were used to probe the samples. The sample-to-detector distance was fixed at $1 \mathrm{~m}$. Scattering data were collected using a Pilatus $300 \mathrm{~K}$ detector with pixel size $172 \times 172 \mu \mathrm{m}$ using a q-range situated in the interval $0.13 \mathrm{~nm}^{-1}<\mathrm{q}<4.7 \mathrm{~nm}^{-1}$ $(q=4 \pi \sin \theta / \lambda)$. All experiments were performed at $25^{\circ} \mathrm{C}$. The data were automatically normalized by the beamline flux and scattering from solutions was subtracted using the beamline software. Absolute scale calibration was performed using water scattering after subtraction of empty cell scattering. ${ }^{1}$ The number of counts at $\mathrm{q}=0$ was calculated by extrapolating a linear fit performed over the flat scattering region. The differential scattering cross-section for pure water at room temperature, $\mathrm{I}_{0}=1.65 \times 10^{-2} \mathrm{~cm}^{-1}\left(\mathrm{I}_{0}\right.$ is the scattering intensity at $\mathrm{q}=0$ ), was used for converting counts into $\mathrm{cm}^{-1}$. Further data treatment was performed with the SASFit package. ${ }^{21}$ Shape models available in the SASFit library were combined to fit the data through a least-squares routine embodied in the code of the program. Data were fitted using linear combinations of two form factors: one for describing micelle contributions (from spherical or ellipsoidal shells) and another to account for fractal aggregates. A flat background was added in the model to account for incoherent scattering and the electronic noise of the detector. 
Fluorescence spectroscopy was performed to follow the interaction between PTA and SDS. Pyrene was used as a probe and the ratio between first and third vibronic emission bands of pyrene was used to determine the micropolarity of the media. ${ }^{19}$ Pyrene in acetone $\left(1 \times 10^{-6} \mathrm{~mol} \mathrm{~L}^{-1}, 20 \mu \mathrm{L}\right)$ was evaporated and $10 \mathrm{~mL}$ volumes of the mixtures were added to produce systems containing $20 \times 10^{-7} \mathrm{mmol} \mathrm{L}^{-1}$ pyrene, which were shaken for $6 \mathrm{~h}$. The spectra were obtained in a Fluoromax-4 (Horiba, Jobin Yvon) with $\lambda_{\text {excitation }}=336 \mathrm{~nm}$, $2 \mathrm{~nm}$ slit at $25{ }^{\circ} \mathrm{C}$. The emitted light was collected in the range of 350 to $450 \mathrm{~nm}$.

The suppressive effect of PTA on pyrene fluorescence was characterized in $\mathrm{NaCl} /$ ethanol/water and $\mathrm{SDS} / \mathrm{NaCl} /$ ethanol/water systems. Aliquots of PTA were added to the prepared systems and the emission spectra, after each addition, were obtained in the same region described above.

\section{Tungsten oxide film preparation and characterization}

Films of the system containing the precursor were obtained and the morphology characterized by scanning electron microscopy (SEM). Silicon (100), coated with a $50-\mathrm{nm}$ silicon oxide layer, was used as substrate $\left(\mathrm{SiO}_{2} / \mathrm{Si}\right)$ of the tungsten oxide films. Prior to film preparation, the substrates were immersed in nitric acid $(10 \% \mathrm{v} / \mathrm{v})$ for $15 \mathrm{~min}$, subsequently rinsed with distilled water and dried at $120{ }^{\circ} \mathrm{C}$ for $30 \mathrm{~min}$. The aqueous systems containing PTA were spin coated onto $\mathrm{SiO}_{2} / \mathrm{Si}$ substrates ( $1500 \mathrm{rpm}, 30 \mathrm{~s}$ ) and heated at $80{ }^{\circ} \mathrm{C}$ for $30 \mathrm{~min}$. This procedure aimed at avoiding damage to films due to strong water release during the calcination process.

The films were calcined under atmospheric pressure in air to remove organics and residual water. The films were placed at center of the furnace and heated from 25 to $500{ }^{\circ} \mathrm{C}$ with $5{ }^{\circ} \mathrm{C} \mathrm{min}^{-1}$ heating rate. The temperature was maintained at $500{ }^{\circ} \mathrm{C}$ for $2 \mathrm{~h}$ and then cooled to room temperature. Morphology of the tungsten oxide films, after calcination, was characterized by SEM, performed with an EVO-50HV-WDS (Carl Zeiss) microscope, at $15 \mathrm{kV}$. A 50-nm thick carbon layer was deposited on the films surfaces by sputtering to improve electrical conductivity. The micrographs were analyzed with Image ${ }^{\mathrm{TM}}$ software. ${ }^{22}$

\section{Results and Discussion}

Physicochemical characterization of the aqueous systems

In this section, the results of conductivity, $\mathrm{pH}, \mathrm{SAXS}$ and fluorescence spectroscopy of the precursor/structuring system composed of PTA/SDS/NaCl/ethanol/water will be discussed in relation to model systems containing SDS/ethanol/water and SDS/NaCl/ethanol/water.

The conductivity measurements of the systems SDS/ethanol/water, SDS/NaCl/ethanol/water and PTA/SDS/NaCl/ethanol/water, as a function of SDS concentration, allowed the structuration process to be followed and the interactions of tungsten oxide precursor (PTA) with the medium to be evaluated. The values of conductivity enabled the critical micellar concentration (CMC) to be determined (Figures 1 and 2), estimated from the intersection between two straight lines obtained by linear regression. The two different straight lines describe the behavior of the systems before and after formation of aggregates. Alternatively, the CMC could be obtained through the Carpena et al. ${ }^{23}$ method, but this method did not present satisfactory results for the studied systems. In Figure 1, it can be observed that for the system SDS/ethanol/water, in low SDS concentrations, the increase in surfactant concentration contributed to a significant increase in conductivity. However, at concentrations higher than the CMC, the formation of micelles led to a decrease in mobility of ions in solution and the conductivity grew slowly with surfactant concentration. The value of CMC found for the SDS/ethanol/water system was $8.69 \mathrm{mmol} \mathrm{L}^{-1}$, a value higher than that found when only water was used as the solvent $\left(8.2 \mathrm{mmol} \mathrm{L}^{-1}\right){ }^{24}$ This result is understandable because ethanol has a lower dielectric constant than water and contributes to reducing the dielectric constant of the medium. The literature ${ }^{24}$ reports that, at low ethanol concentrations, the alcohol molecules interact, mainly with the polar zone of SDS molecules, causing a decrease in electrostatic repulsions of the surfactant polar groups. On the other hand, at higher concentrations of alcohol, electrostatic repulsion between head groups increases, which hinders the process of aggregation of SDS and increases the value of the CMC. ${ }^{25,26}$ In addition, at higher concentrations of ethanol, their nonpolar regions interact with the hydrophobic regions of the surfactant, stabilizing SDS in water and delaying the onset of micellization.

Figure 2 shows results for conductivity as a function of SDS concentration for SDS/NaCl/ethanol/water and $\mathrm{PTA} / \mathrm{SDS} / \mathrm{NaCl} /$ ethanol/water. The insert shows in detail the region of low SDS concentration. The addition of PTA produced a slight increase in conductivity of the media when compared to the system containing SDS/NaCl/ ethanol/water only.

At low concentrations of SDS it was possible to observe a sudden increase in the conductivity values with the increase of surfactant concentration. Above a certain concentration, the value slightly decreased, passing through a minimum, increasing again at higher SDS 


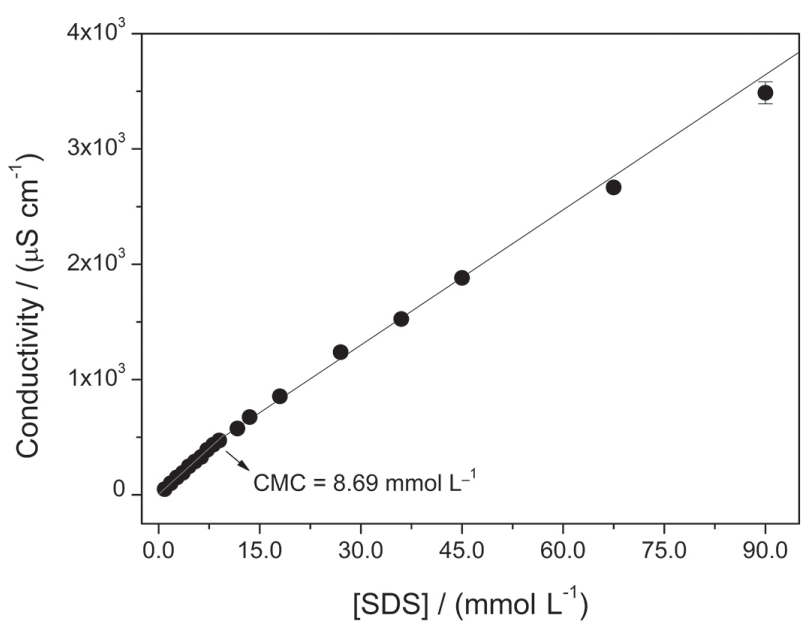

Figure 1. Conductivity of the system SDS/ethanol/water as a function of SDS concentration at $25^{\circ} \mathrm{C}$.

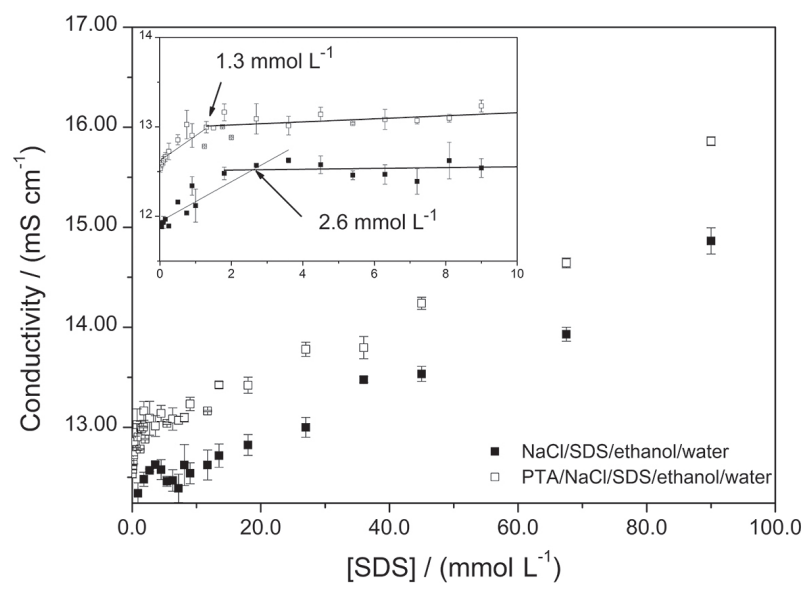

Figure 2. Conductivity as a function of SDS concentration for SDS/ $\mathrm{NaCl} /$ ethanol/water and PTA/SDS/NaCl/ethanol/water systems, at $25^{\circ} \mathrm{C}$. Insert: the same systems shown in region up to $10 \mathrm{mmol} \mathrm{L}^{-1} \mathrm{SDS}$.

concentrations. The presence of $\mathrm{NaCl}$ in both systems is responsible for the hydrophobic repulsion suffered by the surfactant hydrocarbon chains which favors the formation of the first micelles. The addition of salt also promotes a decrease in effective polar area of the surfactant groups, reducing electrostatic repulsion between the micelles, which also favors the aggregation process. ${ }^{27,28}$ The CMC values on Table 1 can be explained by the high polar environment promoted by sodium chloride and hydrogen from peroxotungstic acid, favoring micellization process. The free surfactant chains, in equilibrium with the micelles, are stabilized by the solvent, which reduces their mobility and, thus, explains the small reduction of conductivity observed with the increase of SDS concentration. With further increase in surfactant concentration, dissociation of SDS contributes to a further increase in conductivity.

The conductivity measurements provided important physicochemical parameters related to the micellar interface, such as degree of micelle ionization $(\alpha)$, degree of binding between counter ion and micelle $(\beta)$ and the micellization Gibbs energy $\left(\Delta \mathrm{G}_{\mathrm{m}}\right)$. The degree of micelle ionization $(\alpha)$ was obtained from the ratio $S_{2} / S_{1} . S_{1}$ and $\mathrm{S}_{2}$ are the angular coefficients, obtained by fitting the experimental conductivity data plotted as a function of surfactant concentration, below and above CMC, respectively. The $\alpha$ value is used to calculate $\beta$, according to equation 1:

$\beta=(1-\alpha)$

The value of $\Delta \mathrm{G}_{\mathrm{m}}$ informs the stability of surfactant in the form of aggregates with respect to free monomers in solution, ${ }^{20}$ which, for a conventional surfactant (with an ionizable group), is given by equation 2 :

$\Delta \mathrm{G}_{\mathrm{m}}=\mathrm{RT}(1+\beta) \ln \mathrm{a}_{ \pm}$

where $\mathrm{R}$ is the gas constant, $\mathrm{T}$ is temperature of the experiment and $\mathrm{a}_{ \pm}$is surfactant activity, which for diluted solutions depends on molar fraction of SDS (at CMC) and on other components of the system. The average surfactant ionic activity was calculated by using SDS molar fraction (at CMC) and average ionic activity coefficient, which was obtained by applying the Debye-Hückel Limiting Law. To calculate ionic strength, degree of ionization of PTA was determined by the ratio between its molar conductivity at $10 \mathrm{mmol} \mathrm{L}{ }^{-1}$ PTA aqueous solution (in PTA/SDS/ $/ \mathrm{NaCl} /$ ethanol/water system) and the molar conductivity at infinite dilution. The molar conductivity at infinite dilution was obtained by Kohlrausch's law of independent ionic

Table 1. Thermodynamic parameters obtained through conductivity measurements of SDS/ethanol/water, SDS/NaCl/ethanol/water and PTA/SDS/NaCl/ ethanol/water systems at $25^{\circ} \mathrm{C}$

\begin{tabular}{lccccc}
\hline System & $\alpha$ & $\beta$ & ${\mathrm{CMC} /\left(\mathrm{mmol} \mathrm{L}^{-1}\right)}$ & $\Delta \mathrm{G}_{\mathrm{m}} /\left(\mathrm{kJ} \mathrm{mol}^{-1}\right)$ & $\mathrm{a}_{ \pm}(\mathrm{SDS})^{\mathrm{a}}$ \\
\hline SDS/ethanol/water & 0.73 & 0.27 & $8.67 \pm 0.90$ & $-26.7 \pm 0.2$ & $2.09 \times 10^{-4}$ \\
SDS/NaCl/ethanol/water & 0.12 & 0.88 & $2.60 \pm 0.20$ & $-47.9 \pm 0.5$ & $3.36 \times 10^{-5}$ \\
PTA/SDS/NaCl/ethanol/water & 0.08 & 0.92 & $1.30 \pm 0.10$ & $-52.4 \pm 0.4$ & $1.65 \times 10^{-5}$ \\
\hline
\end{tabular}

${ }^{a} a_{ \pm}$is the SDS average ionic activity when its concentration is equal to critical micellar concentration (CMC). SDS: sodium dodecyl sulfate; PTA: peroxotungstic acid. 
migration, calculated using the infinite dilution conductivity of tungstate ions, hydrochloric acid and sodium chloride.

Table 1 displays the thermodynamic parameters related to SDS/ethanol/water, SDS/NaCl/ethanol/water and $\mathrm{PTA} / \mathrm{SDS} / \mathrm{NaCl} /$ ethanol/water systems. The addition of sodium chloride caused a reduction in $\Delta \mathrm{G}_{\mathrm{m}}$ value, when compared to their $\mathrm{NaCl}$-free counterparts, in accordance with the previous discussion. An increase of ionic strength is responsible for decreasing the SDS ionization degree in $\mathrm{SDS} / \mathrm{NaCl} /$ ethanol/water and PTA/SDS/NaCl/ethanol/ water systems. A reduction in Gibbs energy was higher in the presence of PTA, denoting its influence on stabilization of SDS micelles. Table 1 also shows a decrease in $\alpha$ when PTA was added (and a consequent increase of the coefficient $\beta$ ). The increase in coefficient $\beta$ along with the decrease of $\Delta \mathrm{G}_{\mathrm{m}}$ can be explained by the interaction of sulfate groups with empty d-orbitals of tungsten atoms in PTA.

Figure 3 depicts the surface tension values as a function of SDS concentration for the three systems. The surface tension of the solvent system is significantly lower than that of pure water at $25^{\circ} \mathrm{C}$, reaching $45.3 \mathrm{mN} \mathrm{m}^{-1}$. The use of ethanol reduces the Hansen parameter of hydrogen bonding of the solvent system as a whole (Figure 3a), decreasing the contribution of hydrogen bonding ${ }^{29}$ towards cohesive energy density, and consequently reducing surface tension. When $\mathrm{NaCl}$ was added to the system, a subsequent decrease in the value of surface tension to $37.3 \mathrm{mN} \mathrm{m}^{-1}$ was observed (Figure 3b). In the $\mathrm{SDS} / \mathrm{NaCl} /$ ethanol/water system, sodium chloride increases polarity of the medium and, therefore, alcohol molecules begin to organize preferentially on the water surface, reducing the surface tension of the medium. In the system containing PTA and $\mathrm{NaCl}$ a slight increase in surface tension occurred, to $37.7 \mathrm{mN} \mathrm{m}^{-1}$, when compared to its PTA-free counterparts, caused by the hydrogen interactions of acid with water (Figure 3c). In all systems analyzed, it was possible to observe a reduction in surface tension values with increased SDS concentration. When surfactant concentration is small, the same is preferentially placed at the water/air interface, contributing to minimization of repulsion between hydrophobic groups and water, favoring a significant reduction of surface tension. Subsequent additions of surfactant are dispersed throughout the solution, with no additional effect on the surface tension observed previously, and its value remains constant. The critical micellar concentration of the SDS/ethanol/water system, $8.67 \mathrm{mmol} \mathrm{L}^{-1} \mathrm{SDS}$, could be determined unequivocally through the intersection of linear fitting of the experimental data.

The CMC values obtained for SDS/ethanol/water, SDS/NaCl/ethanol/water and PTA/SDS/NaCl/ethanol/

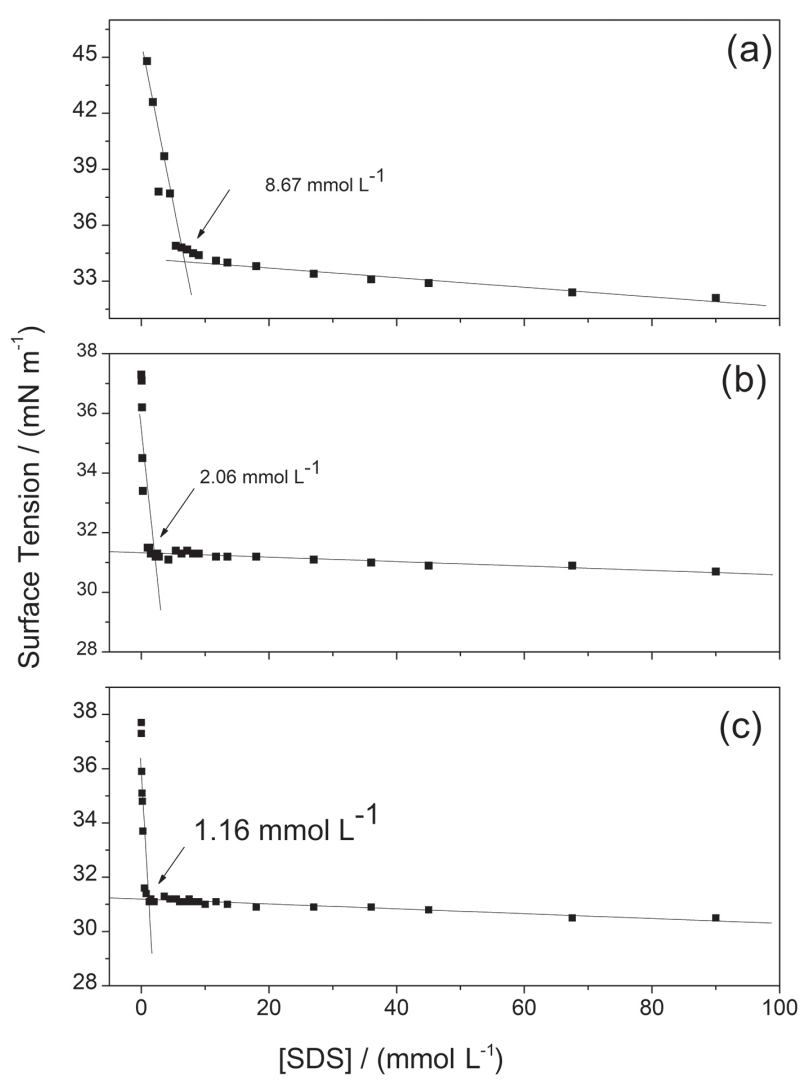

Figure 3. Surface tension as a function of SDS concentration for (a) SDS/ ethanol/water; (b) SDS/NaCl/ethanol/water and (c) PTA/SDS/NaCl/ ethanol/water, at $25^{\circ} \mathrm{C}$.

water systems were $8.67,2.06$ and $1.16 \mathrm{mmol} \mathrm{L}^{-1}$, respectively. These values agree with those values obtained through conductivity measurements.

Information on the nanoscopic structure of the systems, either with or without PTA, was obtained from in situ SAXS experiments. These assays allowed us to probe in detail the organization of the systems under hydrated conditions, and thus obtain insights into precursory structures leading, after calcination of the deposited films, to the formation of larger fractal structures observed in electron microscopy images (see "Morphological characterization of tungsten oxide films" sub-section). In Figure 4, scattering profiles from samples containing SDS, either in the absence (4a) or presence of PTA (4b), are displayed. It is observed that both SDS concentration and PTA availability strongly influence the profile of the curves, demonstrating that the nanoscopic shapes of particles are affected by the conditions of the solution. At intermediate and high q-range $\left(\mathrm{q}>0.4 \mathrm{~nm}^{-1}\right)$, the curves are characterized by a broad peak centered at q ca. $1.8 \mathrm{~nm}^{-1}$, whose intensity increases upon SDS concentration in the sample. The presence of such scattering pattern, with a diffuse band flanked by well-defined minima, is especially noticeable at high SDS concentrations, and it indicates the presence 
of monodisperse shapes in the medium. ${ }^{30}$ On the other hand, the low q-range of the curves is characterized by upturns with rapid increase of scattering intensity. Such behavior at the small-angle region indicates the presence of larger structures in coexistence with smaller monodisperse particles in solution. ${ }^{31}$

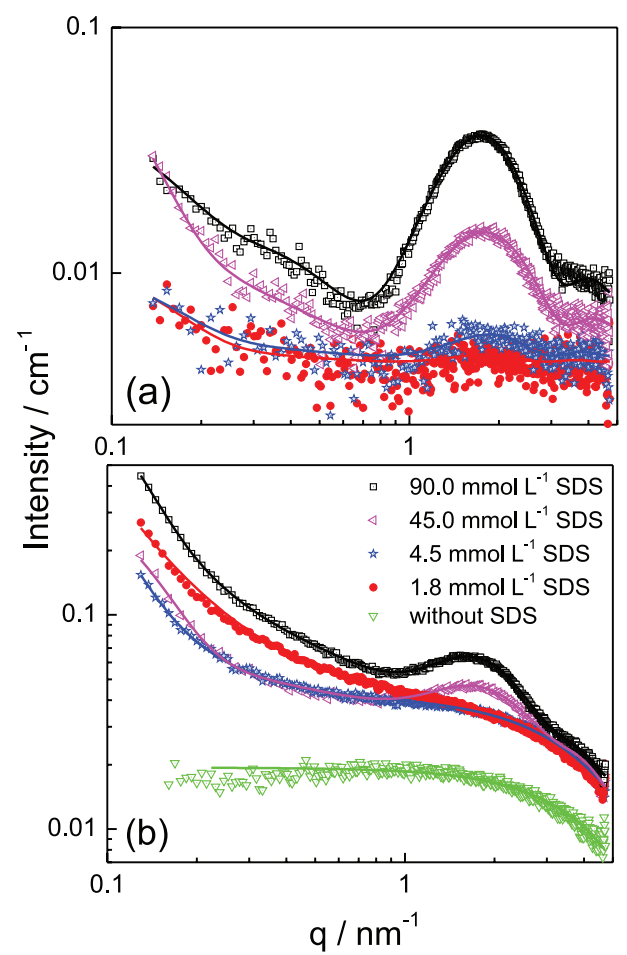

Figure 4. SAXS data from (a) SDS/NaCl/ethanol/water and (b) PTA/SDS/ $\mathrm{NaCl} /$ ethanol/water. Solid lines correspond to model fits performed using a combination of core-shell and mass fractal form factors.

To extract quantitative information on the shape of particles, model fitting was performed using SASFit software. ${ }^{21}$ In Figure 4a, data from samples with $\mathrm{SDS} / \mathrm{NaCl} /$ ethanol/ $\mathrm{H}_{2} \mathrm{O}$ up to $90 \mathrm{mmol} \mathrm{L}{ }^{-1} \mathrm{SDS}$ could be properly fitted using a combination of a mass fractal form factor ${ }^{30,32}$ to describe the low q-region, plus a sphere-shell form factor to account for SDS micelles in solution. ${ }^{33}$ The best fitting parameters are summarized in Table 2. Our fits provided micelle core radii varying between 1.60 and $1.8 \mathrm{~nm}$, in good agreement with the size of extended SDS dodecyl chains $(1.67 \mathrm{~nm}))^{33,34}$ The volume of SDS molecule was computed at $0.47 \mathrm{~nm}^{3}$ from geometrical calculations using its molecular weight $\left(\mathrm{M}_{\mathrm{w}}=288.37 \mathrm{~g} \mathrm{~mol}^{-1}\right)$ and specific mass $\left(\rho=1.01 \mathrm{~g} \mathrm{~cm}^{-3}\right)$. From the volume of spherical micelles revealed SAXS parameters derived above, the aggregation number could be determined ( $\mathrm{N}_{\text {agg }}$ ca. 51), in agreement with SDS into $\mathrm{NaCl}$ solutions. ${ }^{33}$ Similarly, shell thicknesses were found to be between 0.38 and $0.60 \mathrm{~nm}$, also consistent with previous literature. ${ }^{34}$ At higher concentrations, the micelle component was fitted using a prolate spheroid-shell form factor (aspect ratio $>1$ ). ${ }^{34}$ In this case, equatorial semi-axes of micelle hydrophobic cores were found at 1.40 and $1.46 \mathrm{~nm}$ for solutions prepared at 45 and $90 \mathrm{mmol} \mathrm{L}^{-1} \mathrm{SDS}$, respectively. The major semi-axes, aspect ratio $(v) \times$ equatorial semi-axis, were found at 2.64 and $2.8 \mathrm{~nm}$, whereas polar shells exhibited thicknesses $(\Delta \mathrm{R})$ around $0.5 \mathrm{~nm}$. Therefore, we conclude that, at higher SDS concentrations, samples are populated by ellipsoidal micelles with equatorial diameters of ca. $3.9 \mathrm{~nm}$ and longitudinal lengths ca. $6.54 \mathrm{~nm}$. At low q-range, the data fit performed with the mass fractal form factor revealed the presence of coexisting particles with gyration radii $\left(\mathrm{R}_{\mathrm{g}}\right)$ varying from $12.1 \mathrm{~nm}$, at $1.8 \mathrm{mmol} \mathrm{L}^{-1} \mathrm{SDS}$, up to $18.3 \mathrm{~nm}$ for solutions prepared at $90 \mathrm{mmol} \mathrm{L}^{-1} \mathrm{SDS}$. This component is ascribed to the clustering formed by SDS micelles, which have fractal geometry. ${ }^{35}$ Dimensionalities derived from the fits are situated around D ca. 2.6, consistent with compact networks exhibiting ramifications in three coordinates. ${ }^{35}$

Table 2. Best fitting parameters from least-square adjustments on SAXS data

\begin{tabular}{|c|c|c|c|c|c|c|}
\hline & \multirow{2}{*}{$\begin{array}{c}{[\mathrm{SDS}] /} \\
\left(\mathrm{mmol} \mathrm{L}^{-1}\right)\end{array}$} & \multicolumn{3}{|c|}{ Micelle component } & \multicolumn{2}{|c|}{$\begin{array}{l}\text { Fractal } \\
\text { component }\end{array}$} \\
\hline & & $\mathrm{R} / \mathrm{nm}$ & $v$ & $\Delta \mathrm{R} / \mathrm{nm}$ & $\mathrm{R}_{\mathrm{g}} / \mathrm{nm}$ & $\mathrm{D}$ \\
\hline \multirow{5}{*}{ 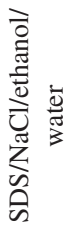 } & 1.8 & 1.8 & 1 & 0.5 & 12.1 & 2.6 \\
\hline & 4.5 & 1.6 & 1 & 0.6 & 11.5 & 2.6 \\
\hline & 9.0 & 1.6 & 1 & 0.4 & 16.6 & 2.5 \\
\hline & 45.0 & 1.4 & 1.88 & 0.5 & 16.5 & 2.6 \\
\hline & 90.0 & 1.5 & 1.91 & 0.5 & 18.3 & 2.5 \\
\hline \multirow{9}{*}{ 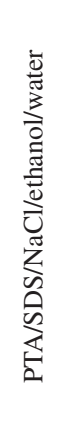 } & 0 & - & - & - & 0.4 & 2.9 \\
\hline & \multirow{2}{*}{1.8} & \multirow{2}{*}{-} & \multirow{2}{*}{-} & \multirow{2}{*}{-} & 25.3 & 1.8 \\
\hline & & & & & 0.5 & 2.9 \\
\hline & \multirow{2}{*}{4.5} & \multirow{2}{*}{-} & \multirow{2}{*}{-} & \multirow{2}{*}{-} & 33.5 & 2.4 \\
\hline & & & & & 0.4 & 2.9 \\
\hline & \multirow{2}{*}{45.0} & \multirow{2}{*}{1.4} & \multirow{2}{*}{2.2} & \multirow{2}{*}{0.7} & 40.7 & 2.6 \\
\hline & & & & & 0.4 & 2.9 \\
\hline & \multirow{2}{*}{90.0} & \multirow{2}{*}{1.3} & \multirow{2}{*}{1.6} & \multirow{2}{*}{1.0} & 41.8 & 2.2 \\
\hline & & & & & 0.5 & 2.9 \\
\hline
\end{tabular}

SDS: sodium dodecyl sulfate; R: equatorial radius of prolate spheroid; v: aspect ratio parameter; $\Delta \mathrm{R}$ : thickness of the hydrophilic micelle shell; $\mathrm{R}_{\mathrm{g}}$ : gyration radius; $\mathrm{D}$ : fractal dimensionality; PTA: peroxotungstic acid.

Data from PTA/SDS/NaCl/ethanol/water mixtures (Figure 4b) were strongly affected by scattering from PTA component. At low surfactant concentrations, 1.8 and $4.5 \mathrm{mmol} \mathrm{L}^{-1}$, contributions from SDS fraction were detectable only at very small angles, in the region carrying information from micelle clusters described above. At the intermediate to high q-range, data were dominated by 
scattering from PTA moieties and the characteristic broad peak from micelle form factor was detected only in samples containing SDS at 45 and $90 \mathrm{mmol} \mathrm{L}^{-1}$. Curves from the more diluted regime could be properly fitted exclusively on the basis of two fractal contributions: one to describe the low q-region assigned to SDS clustering and a second to account for PTA/SDS aggregates in the medium. It should be noted that PTA solutions prepared without SDS did not exhibit the characteristic upturn at low-angle regions (see Figure $4 b$ ); thus, the rise in scattered intensity observed in the PTA/SDS/NaCl/ethanol/water curves in Figure $4 \mathrm{~b}$ could be ascribed to the formation of PTA/SDS aggregates. Interestingly, gyration radii associated to SDS clusters appeared to be systematically higher in PTA-containing samples when compared to their PTA-free counterparts. Indeed, at the highest SDS concentration investigated, the fitting revealed the presence of particles with gyration radius around $41.8 \mathrm{~nm}$, or about twice the size of clusters found in PTA-free solutions. Thus, in light of these findings, we propose that although SDS itself is able to form clusters in solution, PTA enhances aggregation of the surfactant at nanoscale. This result agrees with the thermodynamic data obtained by conductivity (Table 2) and was corroborated by the fluorescence and $\mathrm{pH}$ measurements, which will be shown below.

The $\mathrm{pH}$ values measured for SDS/ethanol/water, $\mathrm{SDS} / \mathrm{NaCl} /$ ethanol/water and PTA/SDS/NaCl/ethanol/ water, as a function of SDS concentration, are shown in Figure 5.

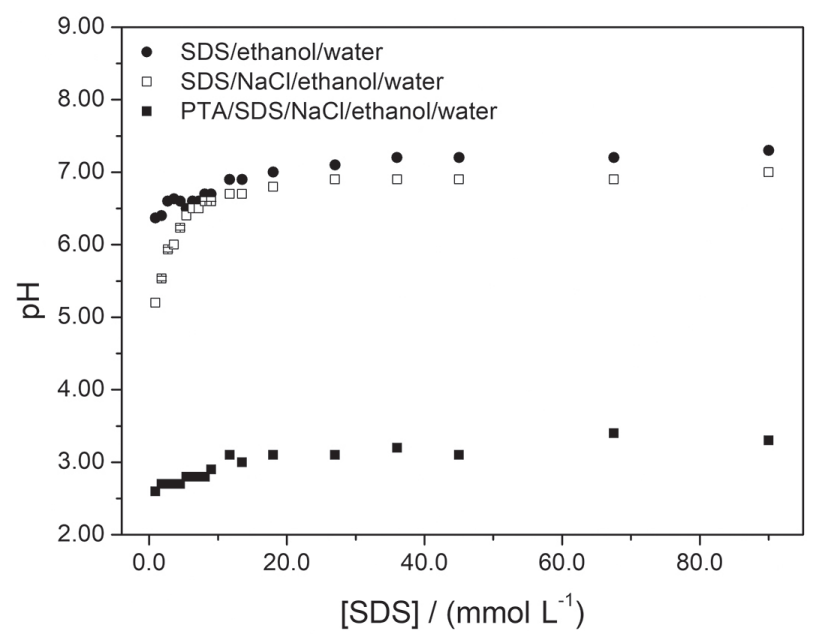

Figure 5. $\mathrm{pH}$ as a function of SDS concentration at $25^{\circ} \mathrm{C}$.

The $\mathrm{pH}$ value of the ethanol/water mixture $(1: 3 \mathrm{v} / \mathrm{v})$ is in agreement with the $\mathrm{p} K_{\mathrm{a}}$ value observed for ethanol, which is 15.5 at $25{ }^{\circ} \mathrm{C}^{36}$ and remained approximately constant with increasing SDS concentration. The addition of salt increases ionic strength and the dielectric constant, promoting partial ethanol ionization, reducing the $\mathrm{pH}$ value; its effect was higher in the region of lower surfactant concentration, compared with the salt-free system. Also, in systems containing salt, it was observed that addition of small quantities of SDS caused a significant increase in $\mathrm{pH}$ values.

The observed stabilization of $\mathrm{pH}$ value in the regime of highest SDS concentration suggests that the surfactant has a buffering effect. In equations 3 and 4 are shown the dissociation of surfactant in water and its buffering effect, respectively.

$\mathrm{RSO}_{4} \mathrm{Na}+\mathrm{H}_{2} \mathrm{O} \rightleftharpoons \mathrm{RSO}_{4(\mathrm{aq})}^{-}+\mathrm{Na}_{(\mathrm{aq})}^{+}$
$\mathrm{RSO}_{4(\mathrm{aq})}^{-}+\mathrm{H}_{(\mathrm{aq})}^{+} \rightleftharpoons \mathrm{RSO}_{4} \mathrm{H}$

In the presence of peroxotungstic acid, $\mathrm{pH}$ decreases to values between 2.6 and 3 , due to acid dissociation (equation 5) over other effects.

$\mathrm{PTA} \rightleftharpoons \mathrm{PTA}_{(\mathrm{aq})}^{-}+\mathrm{H}_{(\mathrm{aq})}^{+}$

The $\mathrm{pH}$ values in the region of lower surfactant concentrations did not present significant changes with addition of SDS, as verified in the SDS/ $\mathrm{NaCl} /$ ethanol/ water system. This behavior can be explained by the fact that, as the surfactant polar group coordinates to the tungsten d-orbitals of PTA (equation 6, sodium ions not shown), the acidic character of PTA decreases relative to PTA in pure water (equation 7, sodium ions not shown). Thus, at concentrations higher than $18 \mathrm{mmol} \mathrm{L}^{-1} \mathrm{SDS}$ (approximately twice the PTA concentration), there was no significant change in acidity of the medium and $\mathrm{pH}$ tended to remain practically constant at values closer to 3.0.

$$
\begin{aligned}
& \mathrm{PTA}+2 \mathrm{RSO}_{4(\mathrm{aq})}^{-} \rightleftharpoons\left[\operatorname{PTA}\left(\mathrm{SO}_{4} \mathrm{R}\right)_{2}\right]^{-2} \\
& {\left[\operatorname{PTA}\left(\mathrm{SO}_{4} \mathrm{R}\right)_{2}\right]^{-2} \rightleftharpoons\left[\operatorname{PTA}\left(\mathrm{SO}_{4} \mathrm{R}\right)_{2}\right]_{(\mathrm{aq})}^{-3}+\mathrm{H}_{(\mathrm{aq})}^{+}}
\end{aligned}
$$

Characteristics of the precursor system, as well as important information on structuring of the precursor/ surfactant system in the solvent medium, were obtained by fluorescence spectroscopy, employing pyrene as probe. Pyrene is a fluorescent probe sensitive to micro polarity of the medium which, when excited at $336 \mathrm{~nm}$, emits in the UV region of the electromagnetic spectrum. The fluorescence spectra of pyrene with the studied systems, in absence and presence of PTA, are shown in Figures 6 and 7 , respectively.

Considering the intensity of pyrene fluorescence bands, it can be observed that they did not change significantly with $\mathrm{SDS}$ concentration in the SDS/ $\mathrm{NaCl} /$ ethanol/water 


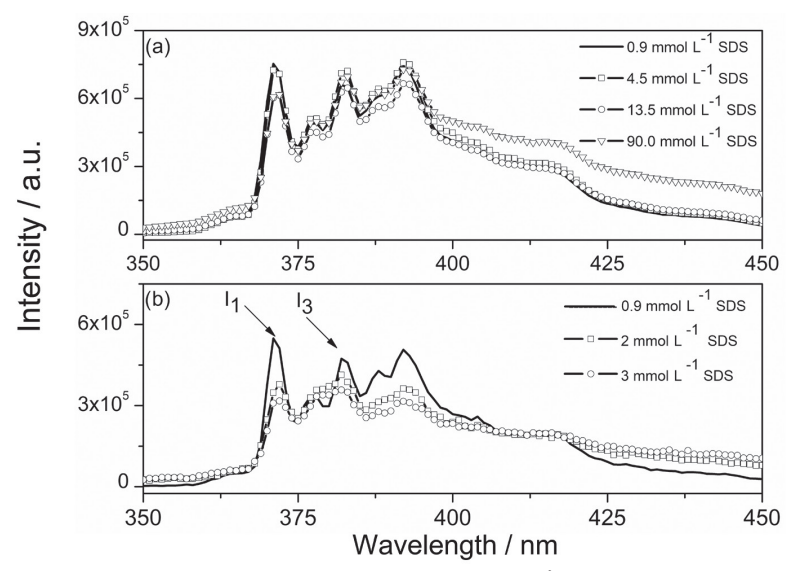

Figure 6. Intensity of emission spectra of pyrene, $\lambda_{\text {exc }}=336 \mathrm{~nm}$, for SDS/ $\mathrm{NaCl} /$ ethanol/water system at different SDS concentrations. (a) Spectra of a wide concentration region; (b) region of concentrations close to the CMC.

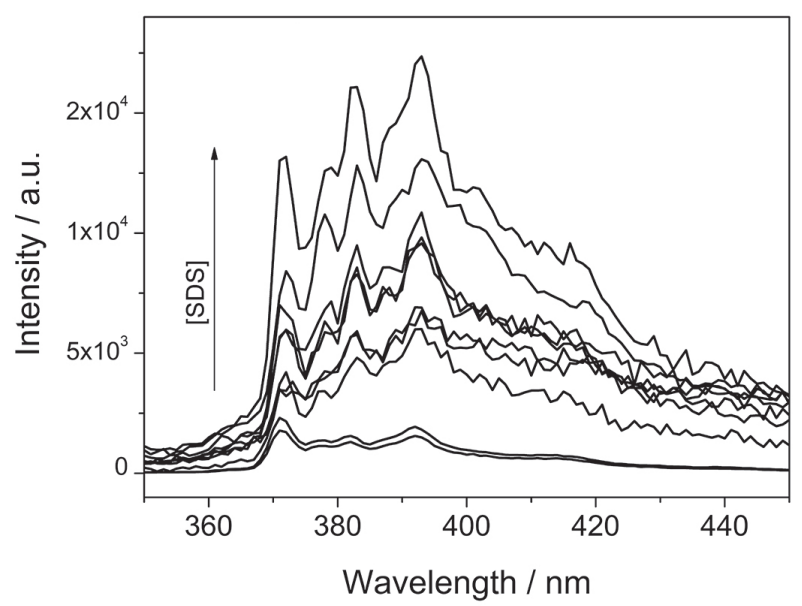

Figure 7. Pyrene emission spectra $\left(\lambda_{\mathrm{exc}}=336 \mathrm{~nm}\right)$ in PTA/SDS $/ \mathrm{NaCl} /$ ethanol/water system at different SDS concentrations.

system (Figure 6a); however, information concerning precursor/surfactant structuration can be obtained through $I_{1} / I_{3}$ ratio, where $I_{1}$ and $I_{3}$ are the intensities of the first $(371 \mathrm{~nm})$ and third $(382 \mathrm{~nm})$ vibronic bands of the pyrene emission spectrum, respectively. ${ }^{37}$ These bands are sensitive to the micro polarity of the media and the inversion of $\mathrm{I}_{1}$ and $\mathrm{I}_{3}$ emission bands in the region of CMC can be observed in Figure $6 \mathrm{~b}$. The presence of PTA in the solvent system, when surfactant concentration is small, resulted in pyrene fluorescence intensity reduction (see Figure 7). It is also observed that the position of the pyrene bands remains unchanged and the $I_{1} / I_{3}$ could be evaluated as shown in Figure 8 .

At concentrations lower than CMC, pyrene ${ }^{37}$ detects the polar environment of ethanol and water molecules. At concentrations higher than $\mathrm{CMC}$, the ratio $\mathrm{I}_{1} / \mathrm{I}_{3}$ diminishes due to the high hydrophobicity of pyrene molecules, which are solubilized inside the micellar phase. In Figure 8a, the values of $I_{1} / I_{3}$ as a function of surfactant concentration

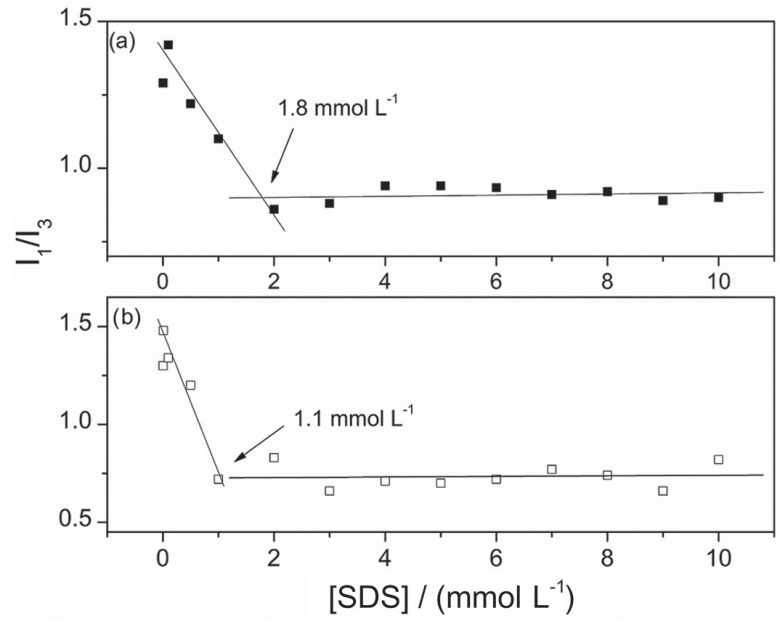

Figure 8. $I_{1} / I_{3}$ ratio of pyrene emission bands as a function of SDS concentration in (a) SDS/NaCl/ethanol/water and (b) PTA/SDS/NaCl/ ethanol/water systems.

are plotted for the SDS/NaCl/ethanol/water system. The ratio $\mathrm{I}_{1} / \mathrm{I}_{3}$, in PTA-free system, gradually decreased with increasing surfactant concentration. The $\mathrm{CMC}$ value found for that system was $1.8 \mathrm{mmol} \mathrm{L}^{-1} \mathrm{SDS}$, close to that obtained by conductivity and surface tension measurements ( 2.6 and $2.06 \mathrm{mmol} \mathrm{L}^{-1}$, respectively).

The $\mathrm{I}_{1} / \mathrm{I}_{3}$ ratio, in presence of peroxotungstic acid, is displayed in Figure 8b. PTA is responsible for the reduction in $\mathrm{CMC}$ of the surfactant. However, we have to consider that in the presence of PTA, a clear determination of CMC can be affected by the suppressive effect of PTA on pyrene emission.

Ghosh et al. ${ }^{38}$ observed a similar effect, with $\mathrm{Cu}^{+2}$ ions as fluorescence suppressors of acridine orange. A possible reason for the decreasing of the intensity could be the radiation scattering by tungsten atoms; however, the addition of SDS and the consequent formation of micelles would contribute also to the scattering of the incident and emitted radiation, resulting in a general lowering of the intensity in the region of analysis $(350-450 \mathrm{~nm})$. On the contrary, the addition of SDS in a PTA/NaCl/ethanol/ water system contributes for the increasing of the emission intensity (Figure 7). In that experiments the pyrene and PTA concentrations remained constant. In the light of these facts, we can affirm that sulfate groups of the surfactant coordinate with PTA overriding the suppressive effect of PTA.

The PTA suppressive effect was investigated in two systems, one composed by $\mathrm{NaCl} /$ ethanol/water, and other containing also $10 \mathrm{mmol} \mathrm{L}^{-1} \mathrm{SDS}(\mathrm{NaCl} / \mathrm{SDS} /$ ethanol/ water), in order to understand the effect of PTA on the pyrene fluorescence. In Figure 9a, the suppressive effect of PTA on the pyrene emission spectra can be clearly observed in $\mathrm{NaCl} /$ ethanol/water system. 

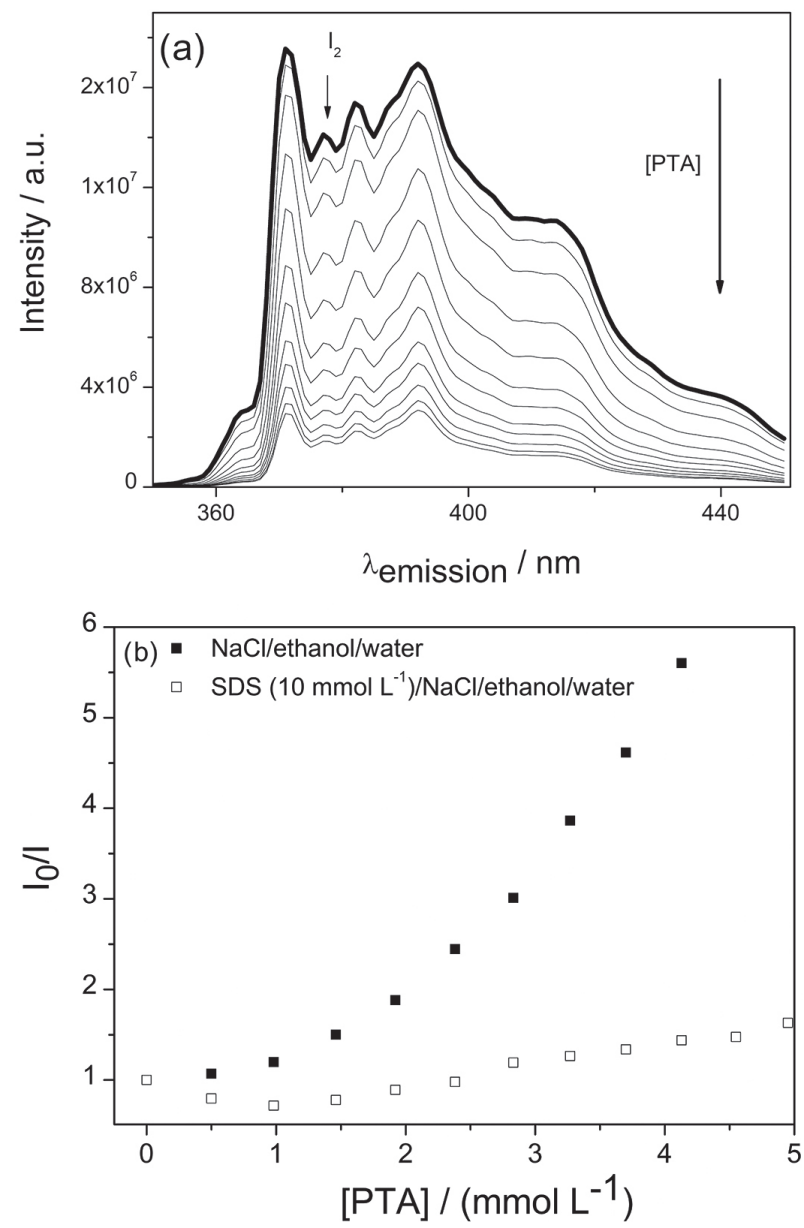

Figure 9. (a) Fluorescence spectra of pyrene present in a system composed of $\mathrm{NaCl} /$ ethanol/water, obtained by addition of $50 \mu \mathrm{L}$ aliquots of PTA stock solution $\left(\lambda_{\text {exc }}=336 \mathrm{~nm}\right)$; (b) ratio between the intensity of pyrene $2^{\text {nd }}$ emission band without PTA $\left(\mathrm{I}_{0}\right)$ and with PTA (I) as a function of PTA concentration.
The ratio $\mathrm{I}_{0} / \mathrm{I}$ is plotted as a function of PTA concentration (Figure $9 b$ ). $I_{0}$ is intensity of the second pyrene emission band measured in the PTA-free system and I is the intensity of the same band in the presence of PTA. We chose this band because it is not influenced by the micropolarity of the medium, as $\mathrm{I}_{1}$ and $\mathrm{I}_{3}$.

Figure 9b clearly shows that PTA acts as fluorescence suppressor and this result agrees with those data obtained in Figures 6 and 7. It also, shows that the pyrene fluorescence increases when surfactant is present. The plots of Figure $9 \mathrm{~b}$ do not obey the Stern-Volmer linear law of collisional quenching, but shows an upward curvature, especially in the case of $\mathrm{NaCl} /$ ethanol/water system. This behavior indicates that both static and dynamic quenching occurs. The collision between the fluorophore, pyrene, and quencher, PTA, during the lifetime of the excited state, characterizes the dynamic collisional quenching. ${ }^{39}$ The static quenching could be due to the formation of non-fluorescent complex between the pi-electrons of pyrene and d-orbitals of tungsten in PTA.

All results discussed above corroborate the nature of the PTA/SDS structuring in aqueous/ethanol medium as resumed in Figure 10.

Figure 10 shows the changes promoted by sodium chloride on the solvation characteristics of the systems (a) favoring the formation of micelles at lower SDS concentration. PTA is coordinated with SDS molecules (b).

\section{Morphological characterization of tungsten oxide films}

Spin coating followed by fast removal of the solvent system restricts the space to two dimensions and permits

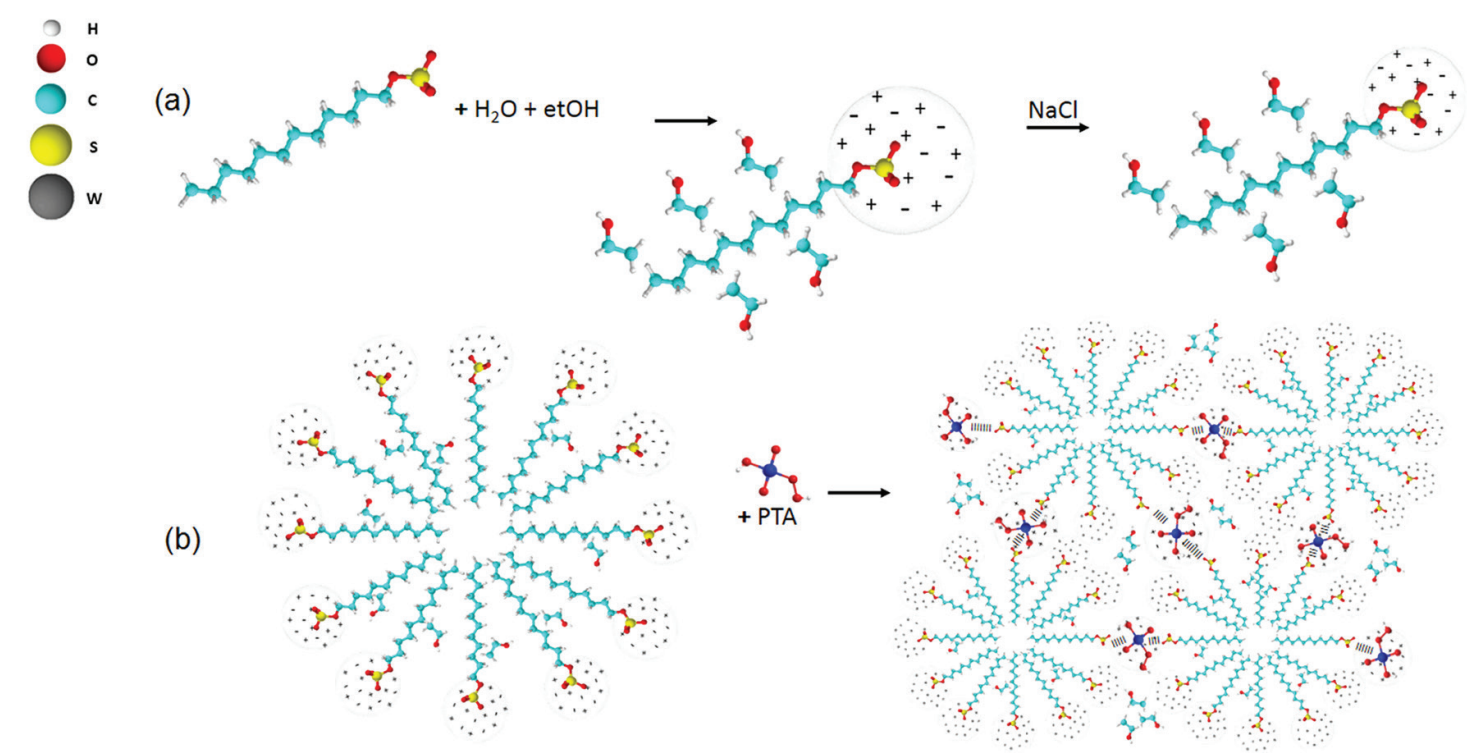

Figure 10. Scheme of the PTA/SDS structure in a medium containing sodium chloride, ethanol and water: (a) sodium chloride and ethanol interact with surfactant chains; the first affects the thickness of the surfactant double layer promoting the aggregation and the second with the hydrocarbon of SDS; (b) PTA, added to the system interacts with the micelles and form the larger aggregates. 
to follow precursor/structuring system in aqueous medium and the morphology of tungsten oxide structures obtained on $\mathrm{SiO}_{2} / \mathrm{Si}$ substrates. Tungsten oxide films were prepared using dispersions of PTA/SDS/NaCl/ethanol/water system. Figure 11 shows a low magnification micrograph of the film obtained with $0.9 \mathrm{mmol} \mathrm{L}^{-1} \mathrm{SDS}$, lower than CMC, in which some tungsten oxide structures forming agglomerates can be observed, but most part of the substrate remains uncovered.

Films obtained from systems with SDS concentrations higher than CMC are shown in Figures 12a-12d.

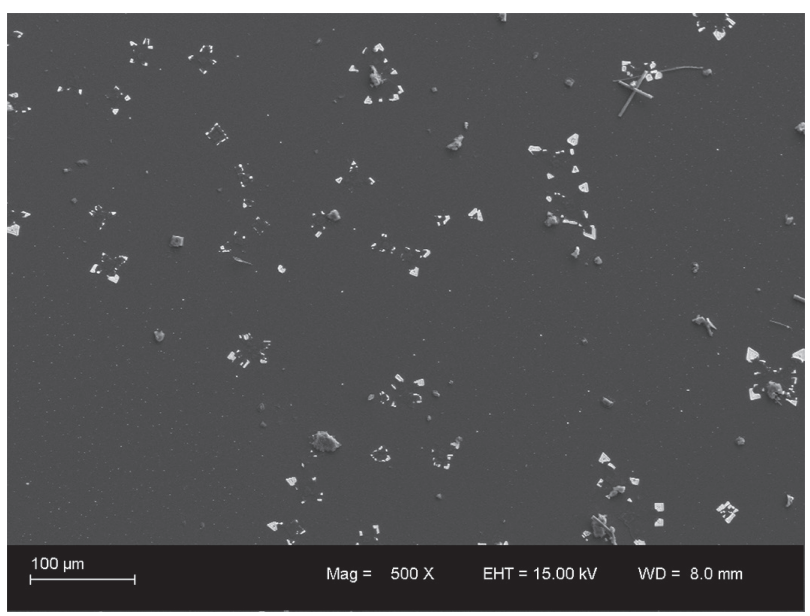

Figure 11. Micrograph of tungsten oxide on $\mathrm{SiO}_{2} / \mathrm{Si}$ substrate obtained from $0.90 \mathrm{mmol} \mathrm{L}^{-1} \mathrm{SDS}$ (lower than CMC), 500× magnification.
All films presented a fractal structure limited by diffusion. The appearance of these structures can be justified by precursor/structuring agent interaction and substrate/suspension interactions in aqueous medium. Such interactions limit Brownian motion of the micellar aggregates, as observed by small-angle X-ray scattering, producing structured oxide after removal of the surfactant by calcination. In addition, it is noted that, in systems containing higher surfactant concentrations, these movements gave rise to structures with a higher fractal dimension, but with a decrease in particle size and, also, a greater coverage of the substrate. These micrometric fractal structures are composed by particles with aspect ratio $1.33 \pm 0.02$ (Figure 13). The particle size distribution, as a function of SDS concentration is shown in Figure $13 \mathrm{~b}$.

Table 3 displays the particle size, as well as the fractal dimension calculated from the micrographs by using FracLac routine for Image J.22

The increase of surfactant concentration is responsible for the decreasing in average particle size. The increase in amount of structuring agent creates a greater number of sites for interaction with PTA, causing reduction in size in the structures observed, and greater distribution on the substrate, resulting in films with a larger fractal dimension. In Figure 14, a comparison of Rg of the fractal structures, obtained by SAXS measurements, with the calculated

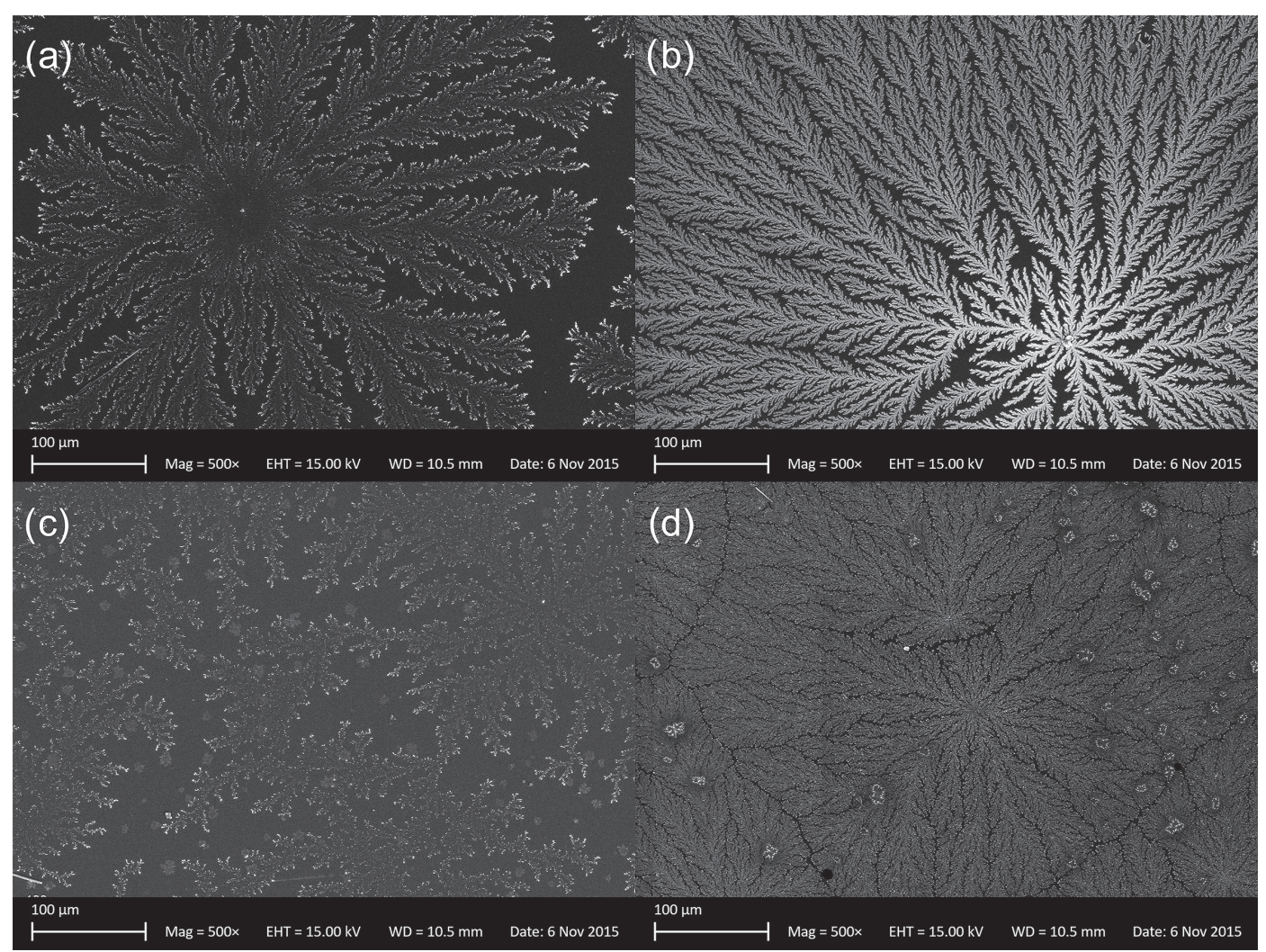

Figure 12. Micrographs of tungsten oxide films at 500 $\times$ magnification, obtained with systems containing: (a) 4.5; (b) 13.5 ; (c) 45.0 and (d) 90.0 mmol L ${ }^{-1}$ SDS. 

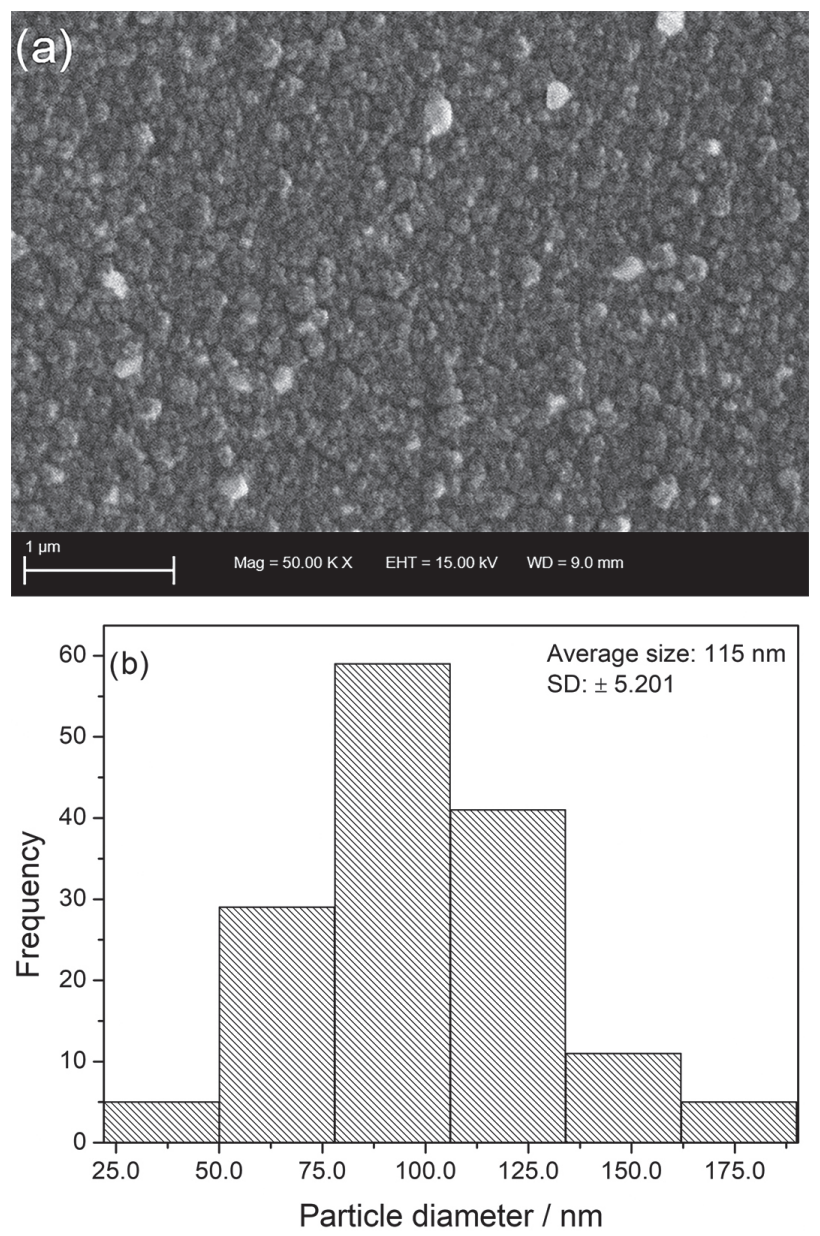

Figure 13. (a) Micrograph of the tungsten oxide films with 50000x magnification and (b) particle size distribution obtained from precursor system containing $13.5 \mathrm{mmol} \mathrm{L}^{-1} \mathrm{SDS}$.

Table 3. Fractal dimension and particle size of tungsten oxide films

\begin{tabular}{lcc}
\hline$[\mathrm{SDS}] /\left(\mathrm{mmol} \mathrm{L}^{-1}\right)$ & Fractal dimension & Particle size / $\mathrm{nm}$ \\
\hline 0.9 & $1.097 \pm 0.022$ & - \\
4.5 & $1.56 \pm 0.01$ & $135 \pm 1$ \\
13.5 & $1.907 \pm 0.006$ & $115 \pm 5$ \\
27.0 & $1.823 \pm 0.007$ & - \\
45.0 & $1.521 \pm 0.007$ & $68 \pm 1$ \\
90.0 & $1.934 \pm 0.002$ & $72 \pm 3$ \\
\hline
\end{tabular}

SDS: sodium dodecyl sulfate.

particle size for the films can be seen. It is observed that increase in dimension of $\mathrm{Rg}$ of the fractals is accompanied by reduction of particle size. This agrees with the previous discussion, since a larger number of SDS molecules keep the precursor molecules isolated. This behavior not only contributes to a reduction in particle size, but also to a reduction in the PTA fluorescence suppressive effect, as discussed in the previous section.

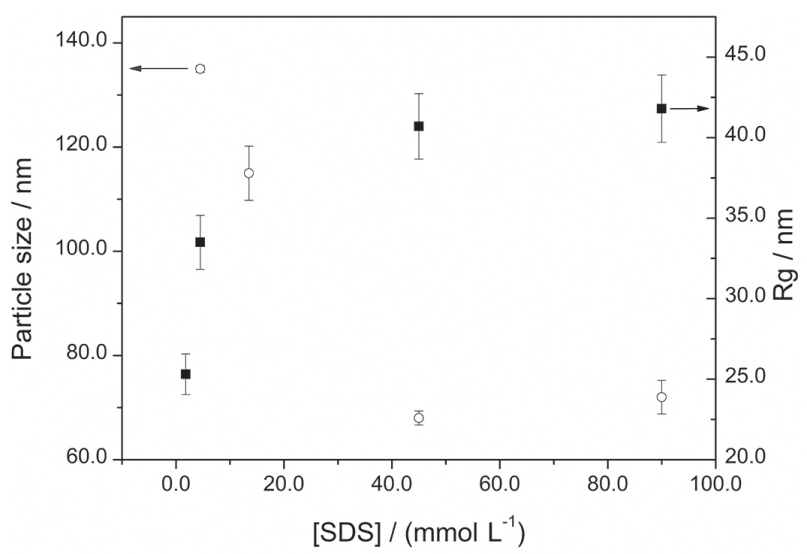

Figure 14. Particle size of tungsten oxide particles and gyration radius of the fractal component as a function of SDS concentration.

The particle sizes after calcination were slightly smaller than those observed in ethanol/water medium by smallangle $\mathrm{X}$-ray scattering.

\section{Conclusions}

In this study, we investigated the nature of morphology development in the preparation of tungsten oxide films from a PTA precursor in ethanol/water medium, in the presence of an architecture-directing agent. We carried out the investigation keeping the ionic strength of the medium constant through addition of sodium chloride. The presence of alcohol favored an increase in CMC and allowed additional stabilization of the micelles in aqueous systems.

The organization of the system containing the precursor was observed by a decrease in surfactant CMC and by formation of fractal aggregates. PTA, in a surfactant free system, promotes a very strong pyrene fluorescence suppression, which can be attributed to a combination of dynamic and static quenching. The collisional effect, as well as the interaction of pyrene pi-electrons interaction with the d-orbitals of tungsten could justify the suppressive behavior of PTA. The increase of pyrene fluorescence intensity with increase of surfactant concentration, together with $\mathrm{pH}$ and Gibbs energy values, allowed us to confirm that there is stabilization of PTA through interaction of empty d-orbitals of tungsten and the sulfate anion of SDS.

The reduction in fractal dimension of the aggregates was obtained by spin coating these aggregates onto $\mathrm{SiO}_{2} / \mathrm{Si}$ surface and further calcination. Films with millimetric fractal structures composed of nanoparticles were observed in systems obtained from SDS concentrations above the CMC. These structures showed a decrease in size with increased concentration of SDS, since a larger number of SDS molecules keeps the precursor molecules isolated. 


\section{Acknowledgments}

The authors thank Coordenação de Aperfeiçoamento de Pessoal de Nível Superior (CAPES), Conselho Nacional de Pesquisa (CNPq, Proc. 311736/2015-7, 307017/2016-8, 311803/2016-4) and São Paulo Research Foundation (FAPESP, 2015/23948-5; 16/24409-3) for financial support. The Laboratório Nacional de Luz Síncrotron (LNLS, Campinas, Brazil) and Laboratório Regional de Nanotecnologia (LRNANO, Porto Alegre, Brazil) are acknowledged for SAXS and MEV measurements.

\section{References}

1. Wang, C.; Gao, Y.; Gao, X.; Wang, H.; Tian, J.; Wang, L.; Zhou, B.; Ye, Z.; Wan, J.; Wen, J.; Sci. Rep. 2016, 6, article ID 35876.

2. Li, H.; Wang, J.; Shi, Q.; Zhang, M.; Hou, C.; Shi, G.; Wang, H.; Zhang, Q.; Li, Y.; Chi, Q.; Appl. Surf. Sci. 2016, 380, 281.

3. Garcia, I. T. S.; Corrêa, D. S.; de Moura, D. S.; Pazinato, J. C. O.; Pereira, M. B.; Nadja, B. D.; Surf. Coat. Technol. 2015, 283, 177.

4. da Costa, N. B. D.; Pazinato, J. C. O.; Sombrio, G.; Pereira, M. B.; Boudinov, H.; Gündel, A.; Moreira, E. C.; Garcia, I. T. S.; Thin Solid Films 2015, 578, 124.

5. Bhosale, N. Y.; Hong, C. K.; Kadam, A. V.; Electrochim. Acta 2017, 246, 1112.

6. Zhou, D.; Che, B.; Kong, J.; Lu, X.; J. Mater. Chem. C 2016 , 4, 8041.

7. Zheng, H.; Ou, J. Z.; Strano, M. S.; Kaner, R. B.; Mitchell, A.; Kalantar-zadeh, K.; Adv. Funct. Mater. 2011, 21, 2175.

8. Wu, C.; Wang, C.; Lin, C.; Wang, S.; Huang, J.; Surf. Coat. Technol. 2013, 231, 403.

9. Yang, B.; Zhang, Y.; Drabarek, E.; Barnes, P. R. F.; Luca, V.; Chem. Mater. 2007, 19, 5664.

10. Bertus, L. M.; Faure, C.; Danine, A.; Labrugere, C.; Campet, G.; Rougier, A.; Duta, A.; Mater. Chem. Phys. 2013, 140, 49.

11. Memar, A.; Phan, C. M.; Tade, M. O.; Appl. Surf. Sci. 2014, 305,760 .

12. Mirtaheri, B.; Shokouhimehr, M.; Beitollahi, A.; J. Sol-Gel Sci. Technol. 2017, 82, 148.

13. Sun, X.; Cao, H.; Liu, Z.; Li, J.; Appl. Surf. Sci. 2009, 255 , 8629.

14. de Moura, D. S.; Pazinato, J. C. O.; Pereira, M. B.; Mertins, O.; Silva, E. R.; Garcia, I. T. S.; J. Mol. Liq. 2018, 269, 92.

15. Wang, W.; Pang, Y.; Hodgson, S. N. B.; J. Sol-Gel Sci. Technol. 2010, 54, 19.
16. Deepa, M.; Srivastava, A. K.; Sood, K. N.; Agnihotry, S. A.; Nanotechnology 2006, 17, 2625.

17. Razzaghi, F.; Debiemme-Chouvy, C.; Pillier, F.; Perrot, H.; Sel, O.; Phys. Chem. Chem. Phys. 2015, 17, 14773.

18. Salmaoui, S.; Sediri, F.; Gharbi, N.; Perruchot, C.; Jouini, M.; Electrochim. Acta 2013, 108, 634.

19. Villetti, M. A.; Bica, C. I. D.; Garcia, I. T. S.; Pereira, F. V.; Ziembowicz, F. I.; Kloster, C. L.; Giacomelli, C.; J. Phys. Chem. B 2011, 115, 5868.

20. Ziembowicz, F. I.; Bender, C. R.; Frizzo, C. P.; Martins, M. A. P.; de Souza, T. D.; Kloster, C. L.; Garcia, I. T. S.; Villetti, M. A.; J. Phys. Chem. B 2017, 121, 8385.

21. Kohlbrecher, J.; Thu, A. F.; J. Appl. Crystallogr. 2015, 48, 1587.

22. Rasband, W. S.; ImageJ; U. S. National Institutes of Health, Bethesda, Maryland, USA, 2017. Available at https://imagej. nih.gov/ij/, accessed in August 2018.

23. Carpena, P.; Aguiar, J.; Ruiz, C. C.; Langmuir 2002, 18, 6054.

24. Wang, X.; Bukusoglu, E.; Abbott, N. L.; Chem. Mater. 2016, $29,53$.

25. Javadian, S.; Gharibi, H.; Sohrabi, B.; Bijanzadeh, H.; J. Mol. Liq. 2008, 137, 74 .

26. Banipal, T. S.; Kaur, H.; Banipal, P. K.; J. Mol. Liq. 2016, 218 , 112.

27. Imperatore, R.; Vitiello, G.; Ciccarelli, D.; D’Errico, G.; J. Solution Chem. 2014, 43, 227.

28. Liu, J.; Zhang, X.; Zhang, H.; J. Chem. Thermodyn. 2014, 72, 1.

29. Yeon, C.; Lee, K.; Lim, J. W.; Carbon 2014, 83, 136.

30. Zemb, T.; Lindner, P.; Neutron, X-Rays and Light. Scattering Methods Applied to Soft Condensed Matter, $1^{\text {st }}$ ed.; Elsevier: North Holland, 2002, p. 541.

31. Pedersen, J. S.; Adv. Colloid Interface Sci. 1997, 70, 171.

32. Teixeira, J.; J. Appl. Cryst. 1988, 21, 781.

33. Jensen, G. V.; Lund, R.; Gummel, J.; Narayanan, T.; Pedersen, J. S.; Angew. Chem., Int. Ed. 2014, 53, 11524.

34. Caetano, W.; Barbosa, L. R. S.; Itri, R.; Tabak, M.; J. Colloid Interface Sci. 2003, 260, 414.

35. Hammouda, B.; J. Chem. Phys. 2010, 133, 084901.

36. Lide, D. R.; Handbook of Chemistry and Physics; CRC Press: Boca Raton, USA, 2005.

37. Piñeiro, L.; Novo, M.; Al-sou, W.; Adv. Colloid Interface Sci. 2015, 215,1 .

38. Ghosh, A. K.; Samanta, A.; Bandyopadhyay, P.; J. Phys. Chem. B 2011, 115, 11823.

39. Lakowicz, J. R.; Principles of Fluorescence Spectroscopy, $3^{\text {rd }}$ ed.; Springer: Maryland, 2006.

Submitted: September 6, 2018

Published online: October 2, 2018 